\title{
Muséologies
}

Les cahiers d'études supérieures

muséologies

\section{Savoir et muséologie : la recherche disciplinaire au musée d'art}

\section{Pierre-Olivier Ouellet}

Volume 1, numéro 2, avril 2007

URI : https://id.erudit.org/iderudit/1033608ar

DOI : https://doi.org/10.7202/1033608ar

Aller au sommaire du numéro

Éditeur(s)

Association Québécoise de Promotion des Recherches Étudiantes en Muséologie (AQPREM)

ISSN

1718-5181 (imprimé)

1929-7815 (numérique)

Découvrir la revue

Citer cet article

Ouellet, P.-O. (2007). Savoir et muséologie : la recherche disciplinaire au musée d'art. Muséologies, 1(2), 52-67. https://doi.org/10.7202/1033608ar

\section{Résumé de l'article}

L'auteur base son étude de la transformation de la fonction de recherche en milieu muséal sur trois facteurs : le contexte postmoderne, l'actuelle muséologie du spectaculaire, puis la conception de l'objet au sein de la discipline muséologique. Cette étude sert de prémisse à l'analyse qu'il propose de la relation entre la recherche et le musée d'art. d'utilisation que vous pouvez consulter en ligne.

https://apropos.erudit.org/fr/usagers/politique-dutilisation/ 


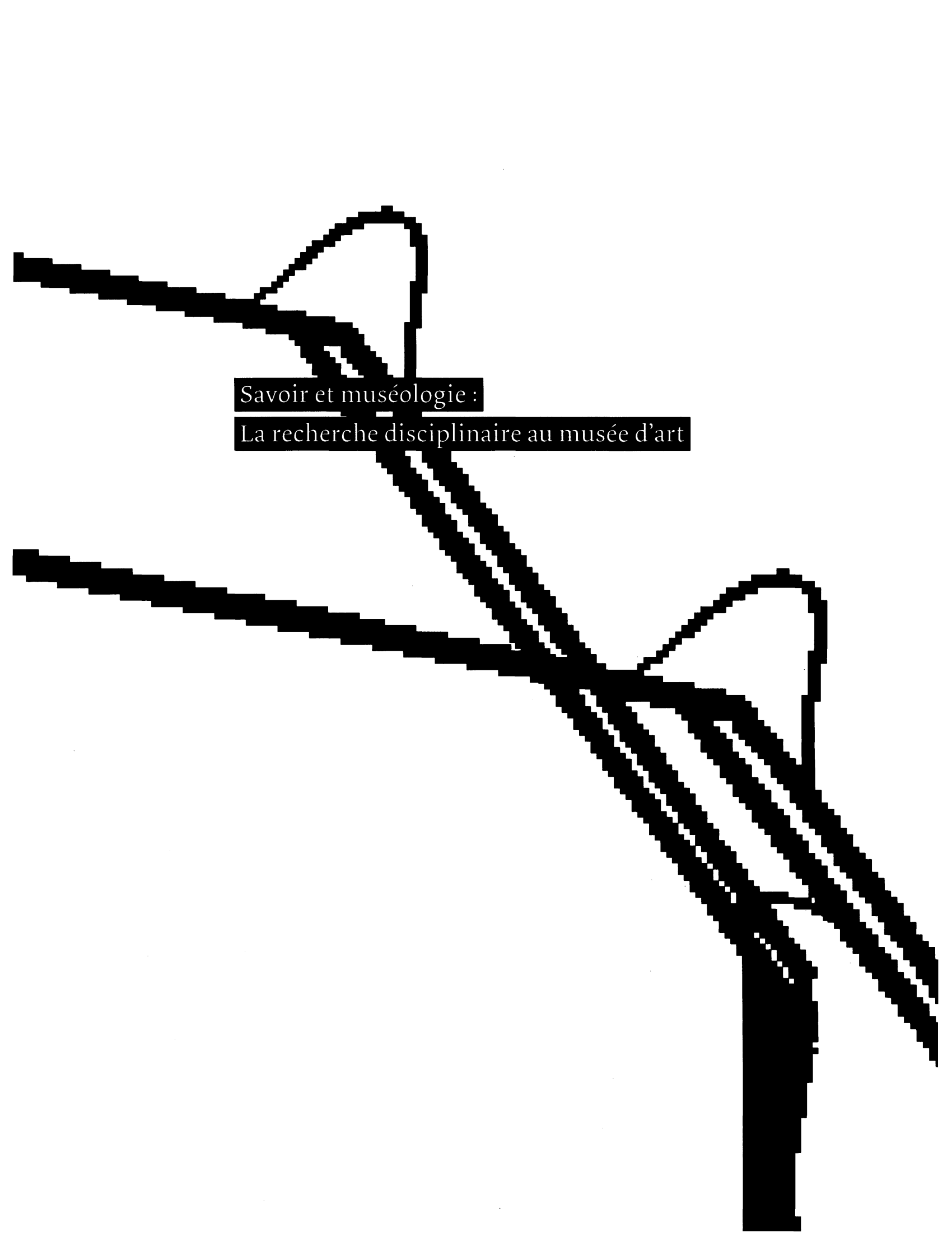


Lauteur base son étude de la transformation de la fonction de recherche en milieu muséal sur trois facteurs : le contexte postmoderne, l'actuelle muséologie du spectaculaire, puis la conception del'objet au sein dela discipline muséologique. Cette étude sert de prémisse à l'analyse qu'il propose de la relation entre la recherche et le musée d'art.

PIERRE-OLIVIER OUELLET EST TITULAIRE DE BACCALAURÉATS EN ARTS VISUELS ET EN HISTOIRE DE L'ART. EN 2004, IL A RÉALISÉ L'EXPOSITION «ART ET DÉVOTION : LES RELIQUAIRES À PAPEROLES», PRÉSENTÉE AU MUSÉE DES HOSPITALIĖRES DE L'HÓTEL-DIEU DE MONTRÉAL, EN PLUS D'EN RÉDIGER LE CATALOGUE. IL A OBTENU, LA MÉME ANNÉE, SA MAîtrise EN MUSÉologie. PIERRE-OLIVIER OUELLET EST ACTUELLEMENT CHARGÉ DE COURS ET ÉTUDIANT AU DOCTORAT EN HISTOIRE DE LART À L'UNIVERSITÉ DU QUÉBEC À MONTRÉAL. SA THĖSE, AMORCÉE EN 2004, TRAITE DES COLLECTIONS D'EUUVES D'ART EN NOUVELLE-FRANCE. 
"Précarité de la recherche".

Musée, vol. $10, n^{\circ} 1$, printemps

1987, p. 12.

[2]

LOCHNAN, Katharine A.

"La recherche : pierre philosophale ». Muse, vol. 1, $n^{\circ} 3$, automne / octobre 1983, p. 17.

[3]

MONTPETIT, Raymond.

"Musées et universités : des fonctions en redéfinition, des missions complémentaires, des collaborations requises".

In. JAUMAIN, Serge (dir.).

Les musées en mouvement : nouvelles conceptions, nouveaux publics. Bruxelles :

Éditions de l'Université de Bruxelles, 2000, p. 43.

[4]

BAZIN, Germain.

Le temps des musées. Liège : Desoer, 1967, p. 144.

SCHIELE, Bernard.

«Les trois temps du patrimoine : note sur le découpage symbolique». In. SCHIELE, Bernard (dir.). Patrimoines et identités. Québec : Musée de la

civilisation, 2002, p. 225.
D'une muséologie de l'objet, axée sur la collection, à une muséologie de société, axée sur les publics et les services, le XXe siècle a été marqué par une réévaluation des fonctions muséales. De fait, le développement des techniques, du marketing et de l'éducation, de même que le positionnement des musées dans l'industrie culturelle, ont métamorphosé l'image de ce temple sacré des muses en un espace à la fois événementiel et plus convivial.

Cette modernisation du musée au sein d'une prise de conscience aiguë envers le public a bien évidemment eu de nombreux effets bénéfiques, notamment par la démocratisation de la culture. Mais, dans la nouvelle répartition du temps, de la mobilisation des ressources et du financement en fonction du public, ainsi que par l'émergence d'une philosophie de l'attraction, les musées n'ont-ils pas sacrifié une fonction effacée ${ }^{(1)}$ comme la recherche sur l'autel du spectaculaire? De plus en plus délaissée par certains musées, elle est bien souvent considérée à court terme comme un luxe $e^{(2)}$, une pratique dont la rentabilité (immédiate) n'est pas assurée.

L'histoire révèle bien, pourtant, la place fondamentale dévolue au développement des connaissances parmi les premières préoccupations rattachées au collectionnement et au musée. De la Renaissance au XVII siècle, le cabinet de curiosités est d'abord un lieu d'étude né d'une volonté d'ordonner les objets, d'en établir les similitudes symboliques ${ }^{(3)}$ et matérielles, dans un univers où se côtoient et se mélangent la pensée aristotélicienne, les découvertes contemporaines et les croyances issues du Moyen-Âge. Dès le XVII siècle, certaines collections, dont on reconnaît les rôles éducatif et scientifique, se greffent aux institutions du savoir. En effet, « institut propre à l'avancement des connaissances humaines, écrivait l'historien de l'art et conservateur du Louvre Germain Bazin, le musée trouvera un berceau naturel au sein de l'Université " "4]. Le cabinet Amerbach est ainsi acquis par la ville de Bâle et exposé dans la bibliothèque universitaire de 1671 à 1849 . De même, l'Ashmolean Museum ouvre ses portes en 1683, constituant un lieu d'études et de référence de l'université d'Oxford. Au Siècle des Lumières, où «l'objectif [scientifique est] le dévoilement de l'intelligibilité du monde grâce à l'exercice sans limites de la raison ${ }^{(5)}$, on procède à la remise en ordre des collections, favorisant les distinctions disciplinaires et, incidemment, 
la spécialisation du savoir. Jusqu'au XX $X^{e}$ siècle, les connaissances demeurent au centre des préoccupations de plusieurs musées fondés au XIX ${ }^{e}$ siècle, tout comme la conservation de l'objet qui en témoigne. Tel que le synthétise Paul Rasse :

Au sein des processus d'élaboration et de communication des savoirs scientifiques, le musée a joué un rôle décisif dans le règne des objets matériels, peut-être aussi important que celui de l'imprimerie dans l'ordre de l'écrit. Il a incontestablement été ce que Bruno Latour appelle « un dispositif de mobilisation des connaissances ", un panoptique, pour reprendre l'expression de Michel Foucault, accumulant les objets de savoir, les rassemblant depuis tous les coins du monde, les classant et les conservant pour les donner à étudier à la communauté scientifique ${ }^{[6]}$.

Au cours du XXe siècle, dans la transformation progressive vers une muséologie de société, même un intellectuel indocile comme GeorgesHenri Rivière, figure emblématique du renouveau muséologique, conçoit pleinement le rôle fondamental de la recherche au musée. Dans ses leçons publiées en 1989, il enseignait que :

La fonction de recherche constitue la base de toutes les activités de l'institution, elle éclaire sa politique de conservation et d'action culturelle. Est-elle déficiente, les autres fonctions s'en ressentent fâcheusement. Doté d'organes de recherche, le grand musée saura s'accorder à l'avancement de sa ou de ses disciplines de bases, selon ses propres moyens d'étude. Affirmer la place fondatrice de la recherche en l'associant à la définition même du musée, c'est dire que le statut et la légitimité de l'institution muséale en dépendent ${ }^{[7]}$.

De fait, les propos de Rivière soulignent l'importance de la recherche

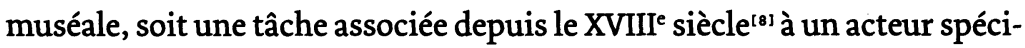
fique du musée : le conservateur. Expert disciplinaire, celui-ci se porte garant de la conservation de l'intégrité matérielle et intellectuelle de la collection, ainsi que de son enrichissement. Plus qu'un figurant, le conservateur est un connaisseur. Il participe aux avancées de la discipline par ses pratiques et ses connaissances acquises tant par sa formation et ses lectures que par la fréquentation et l'examen des objets du musée.

Depuis les années 1980, selon le constat de Raymond Montpetit, la place accordée à la recherche muséale tend toutefois à péricliter ${ }^{[9]}$. Le conservateur effectue dorénavant des tâches de plus en plus associées à la diffusion : " on nous demande, écrit le conservateur Jochen Sander, de faire les choses rapidement, de faire des choses qui marchent et qui attirent le public ${ }^{[10]}$. Empêtré dans un travail de gestion et de coordination, le conservateur doit de plus en plus " prendre sur ses heures de temps libre ou de sommeil le temps

\section{[6]}

RASSE, Paul.

Techniques et cultures au musée : enjeux, ingénierie et communication des musées de société. Lyon : Presses universitaires de Lyon, 1997, p. 152. [7]

RIVIËRE, Georges-Henri. La muséologie selon Georges Henri Rivière : cours de muséologie, textes et témoignages.

Paris : Dunod, 1989, p. 169.

[8]

En 1713, un règlement du Ashmolean Museum d'Oxford prévoit la présence de curatores qui se partagent les collections par spécialité.

(Voir BAZIN, op. cit, p. 145.)

[9]

MONTPETIT,

"Musées et universités... *, op. cit, p. 44.

[10]

SANDER, Jochen.

"Le musée est-il encore un lieu de recherche? * In. GALARD, Jean (dir.). L'avenir des musées. Paris : Réunions des musées nationaux, 2001, p. 356. 
consacré à la recherche " ${ }^{[11]}$. De plus, la figure même du conservateur comme spécialiste et chercheur professionnel est ébranlée. À cet effet, Paul Rasse soutient que «les conservateurs n'ont ni le temps, ni souvent la formation pour entreprendre eux-mêmes des recherches " ${ }^{[12]}$. Le muséologue propose alors de reléguer cette tâche à des équipes pluridisciplinaires et à des universitaires. Le musée serait-il ainsi destiné à ne jouer qu'un rôle d'interface entre un savoir externe et les publics? Nous commencerons par présenter trois facteurs pour esquisser cette transformation soudaine des pratiques et de la conception du rapport entre la recherche et les institutions muséales, soit le contexte postmoderne, l'actuelle muséologie du spectaculaire, puis la conception de l'objet au sein de la discipline muséologique. Après cette première partie, nous délimiterons notre champ d'investigation à l'étude de la relation entre la recherche et le musée d'art dans un champ disciplinaire spécifique, soit l'histoire de l'art.

[11]

LACROIX, Laurier. "La recherche au musée : un enjeu social ». Musée, vol. 10, $\mathrm{n}^{\circ} 1$, printemps 1987, p. 21.

[12] RASSE, op. cit., p. 154.

[13]

MONTPETIT,

"Musées et universités...", op. cit, p. 46.

[14]

BONNY, Yves. Sociologie du temps présent: modernité avancée ou postmodernité? Paris : Armand Colin, 2004, p. 65.

\section{Postmodernité, subjectivité des savoirs}

Depuis les années 1980, l'appropriation subjective et sélective du savoir par les individus, dans une époque postmoderne du " chacun-pour-soi ", soulève la question de l'expertise du conservateur. En effet, les choix d'acquisition et le discours du conservateur sont perçus comme une volonté implacable de conserver un monopole de la parole légitime, de proposer pour tous. Ainsi, on questionne la validité du jugement du conservateur dans la hiérarchisation des valeurs artistiques, lequel sélectionne et "patrimonialise" les objets qui lui semblent exemplaires. À cette critique du poids décisionnel exercé par une seule figure emblématique du savoir de l'institution s'ajoute la question du discours univoque retrouvé dans les catalogues et les écrits d'expositions, à une époque où l'interprétation est dite personnelle, et même plurielle, l'objet étant jugé polysémique.

Parallèlement à cette transformation, comme le mentionne Raymond Montpetit, la «critique épistémologique, de son côté, a tôt fait de montrer que les savoirs disciplinaires (histoire, ethnologie, anthropologie, histoire de l'art) sur lesquels les musées se sont fondés, sont des constructions très datées " ${ }^{[13]}$. Dans cette posture typiquement postmoderne "de rupture, de prise de congé ou de déconstruction critique à l'égard des orientations intellectuelles et culturelles " ${ }^{[14]}$, la nature disciplinaire du musée est questionnée, étant donné l'accumulation d'idées et de savoirs provenant d'une autre époque. 


\section{La muséologie du spectaculaire}

Dans une logique actuelle de subsistance étroitement liée à la fréquentation du musée, attirer le visiteur implique que la gestion des publics devienne prioritaire pour les institutions. La mise en branle d'une véritable stratégie de calcul fait en sorte que dominent les activités de diffusion (l'exposition, l'éducation, les communications), assurant une rentabilité, les résultats des efforts fournis étant visibles en peu de temps. La muséologie du spectaculaire naît alors d'une adaptation au marché. Selon François Mairesse, le spectaculaire muséal est basé sur l'image visuelle (de l'architecture extérieure, de sa signalétique et de sa muséographie), la technique (permettant la rationalisation des activités), le ludique et le politiquement correct, puis

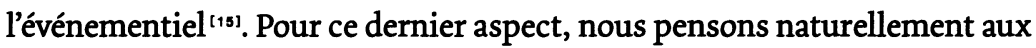
expositions blockbusters où, comme l'écrit Germain Bazin, «la plus belle sculpture du monde a une plus grande valeur d'actualité lorsqu'on la transporte sur un chariot ${ }^{[16]}$. De fait, tel que le précise Mairesse :

Même lorsqu'ils n'ont pas recours à ce genre d'exposition, l'événement n'en demeure pas moins une règle à observer. [...] La raison est assez simple. Pour attirer les visiteurs, les musées ont principalement recours à la reconnaissance médiatique (presse, télévision), laquelle ne fonctionne plus depuis longtemps que par l'événementiel ${ }^{[17]}$.

Créer l'événement, susciter l'engouement des foules et des médias, dynamisent ainsi le musée spectaculaire.

La logique même des dispositifs médiatiques et événementiels inverse la relation traditionnelle à l'objet. Ce n'est pas son passé, ni sa valeur d'exemple pour l'avenir, qui importent, mais son caractère immédiat. Et, pour le visiteur, l'expérience vécue serait plus importante que l'acquisition de connaissances. Comme le propose Bernard Schiele :

C'est la production de connaissances nouvelles qui se trouve maintenant à la remorque de l'innovation. Pourquoi ? Parce que, d'une part, la quête d'intelligibilité s'est muée en vision prométhéenne - il s'agit moins de comprendre le monde que de le transformer -; d'autre part, plus prosaïquement, parce que le maintien du développement économique repose en grande partie sur une relance continuelle de la productionconsommation, laquelle, en retour, exige l'introduction constante de nouvelles idées et de nouveaux produits sur le marché. Autrement dit, l'innovation - pas seulement technologique, mais dans tous les domaines d'activité - présuppose l'obsolescence systématique comme nouvelle force productive. La connaissance y est subordonnée ${ }^{[18]}$.

\section{[15]}

MAIRESSE, François.

Le musée, temple spectaculaire : une histoire du projet muséal. Lyon : Presses universitaires de Lyon, 2002, p. $135-138$.

[16]

BAZIN, op. cit., p. 276.

[7]

MAIRESSE,

op. cit, p. 137.

[18]

SCHIELE, op. cit, p. 225. 
"Principes esthétiques et préparation des expositions didactiques". In. DESVALLÉES, André (dir.). Vagues : une anthologie de la nouvelle muséologie. Mâcon /

Savigny-le-Temple : Éditions W / Muséologie nouvelle et expérimentation sociale, 1992

vol. 1, p. 350

[20]

BARBIER BOUVET

Jean-François. "Le visiteur dans tous ses états".

In. VÉRON, Eliséo et Martine

LEVASSEUR. Ethnographie

de l'exposition : l'espace, le corps et le sens. Paris : Centre Georges Pompidou, 1991, p. 8.

L'auteur y énumère tout ce qui lui semble être un support pour l'exposition, soit le texte,

l'image, les objets et même le son

[21]

DELOCHE, Bernard.

Le musée virtuel : vers une éthique des nouvelles images.

Paris : Presses universitaires de France, 2001, p. 254.

[22]

DESVALLÉES, André «Exhiber ou démontrer? L'objet de l'exposition". Musées, vol. $19, \mathrm{n}^{\circ} 1$ 1997, p. 29.

[23]

Comme le rédige Bernard Schiele (op. cit., p. 233-234), * le passé est obligatoirement mis en scène et offert en spectacle. La vérité, l'authenticité, l'exactitude du spectacle

ne restituent qu'en partie la richesse et la complexité des réseaux de significations qui reliaient l'Autre-sujet ou l'Autreobjet à son monde. [...] Que reste-t-il alors du spectacle du passé? Un modèle, mais un modèle détaché de son contexte. C'est-à-dire une représentation décontextualisée, libre de toute attache, disponible pour une nouvelle interprétation."
Le musée voit non seulement le rapport au savoir se transformer, de sorte que la connaissance devient secondaire, mais il se trouve également, dans sa nature intrinsèque, métamorphosé par le fait qu'il occupe une place reconnue dans l'industrie culturelle.

\section{L'objet muséologique}

La muséologie propose de nombreuses définitions de l'objet muséal. Il est désigné sous le terme d'objet-témoin ${ }^{[19]}$, de support ${ }^{[201}$, de musealia (" objets de musée " ${ }^{[211}$ ), d'artefact ou encore d'expôt (" équivalent de l'un des sens de l'anglais exhibit, pour désigner tout ce qui était exposé " ${ }^{[22]}$ ). L'objet, devenu une entité métaphysique pour des besoins de théorisation, réfere alors à l'objet dépouillé de ses particularités. La distinction primaire entre deux objets de nature différente, par exemple entre l'œuvre d'art et l'épi de maïs, n'existe plus du moment où tous deux ont été «muséalisés». Ainsi, puisque maintenu artificiellement en vie sous vitrine, l'objet est dénaturé. En échange de sa conservation, il est décontextualisé ${ }^{(23)}$, il perd son usage d'origine en même temps qu'il ne sert plus qu'à signifier ${ }^{[24]}$.

Dans l'exposition, l'objet, puisqu'il est décontextualisé, n'est plus qu'un signe enchâssé dans une logique sémiologique, constituant un élément d'un discours établi et proposé dans la production du langage. Comme le propose Jean Davallon, il est « objet d'un monde de langage. Son statut et sa signification seront donc définis par les rapports qu'il entretiendra avec les autres objets de l'exposition " ${ }^{255}$. Autrement dit, et tel que le précise Bernard Schiele, l'objet « est transposé par la mise en exposition dans l'environnement du musée, lequel, de ce fait, substitue sa propre signification à celle qui a été perdue " ${ }^{266}$. L'objet étant jugé polysémique, il est à la fois tout - son contenu étant tributaire d'une adaptation au contexte d'exposition - et rien - «l'objet n'est la vérité de rien du tout " $^{[27]}$ (selon la formule lapidaire de Jacques Hainard) -, son histoire étant souvent évacuée, sa richesse matérielle et son authenticité constituant même des éléments négligeables. Ce rôle de l'objet muséal comme illustration fait en sorte que la collection du musée ne constitue plus qu'un ensemble de moyens, des moyens entreposés dans des réserves, émergeant en fonction de l'actualité et de l'événement.

De ce point de vue, la recherche sur l'objet au musée constitue véritablement un hiatus dans la compréhension de l'institution muséale. Tout d'abord, lobjet, dans sa déclinaison muséologique, ne se rattache plus à une discipline spécifique. Ensuite, l'objet est un signe dont le signifiant dépend de la trame discursive d'une exposition. Puis, l'exposition constituant l'attrait principal du musée orienté vers les publics et le spectaculaire, garants de la survie 
d'une institution, l'objet de collection ne semble plus revêtir qu'un intérêt secondaire, voire tertiaire. La collecte au guichet supplante la collection. Enfin, dans un contexte postmoderne - où la connaissance semble maintenant subordonnée à l'innovation et le savoir, à «l'obsolescence systématique comme nouvelle force productive» -, que devient la recherche muséale?

\section{Et pourtant... la recherche au musée}

À la suite d'une étude menée en 1996 auprès des institutions muséales, François Mairesse révélait que la recherche et la collection, parfois considérées comme des intérêts révolus, constituaient toujours des fonctions prioritaires pour des importants groupes de musées ${ }^{[28}$. Vœu pieux ou déclaration d'intention, le musée peut-il encore représenter un lieu de production de savoirs et de recherches spécialisées? Est-ce que le conservateur joue encore un rôle essentiel dans les recherches? Avant de nous avancer davantage, demandons-nous, a priori, si la volonté muséologique d'homogénéiser les définitions de linstitution, en mettant de côté son aspect disciplinaire, ne viendrait pas embrouiller la réalité muséale ? Pourquoi, en effet, dépouiller le musée de son aspect disciplinaire? Pourtant, comme l'affirme Rivière, la recherche disciplinaire constitue un enjeu central des fonctions muséales :

Doté d'organes de recherche, le grand musée saura s'accorder à l'avancement de sa ou de ses disciplines de bases, selon ses propres moyens d'étude. [...] Ainsi, toute recherche implique à l'origine une réflexion générale sur la situation de la discipline de base et une évaluation des lacunes du savoir dans le domaine considéré. La collecte des objets et spécimens s'effectue en fonction de ces données et des lacunes correspondantes dans les collections. L'acquisition est donc inscrite dans le processus de recherche de musée et devient, de ce fait, acte scientifique ${ }^{\text {(2201 }}$.

Nous proposons donc, pour cette seconde partie, d'effectuer une étude de la fonction de la recherche disciplinaire au musée. Cela dit, pour ce vecteur de recherches ${ }^{[30]}$, nous étudierons plus spécifiquement la relation entre l'histoire de l'art et l'institution muséale au sein du musée d'art.

\section{[24]}

Pomian proposait, à cet effet, le terme de sémiophore, soit * des objets qui n'ont point d'utilité

[...], mais qui représentent l'invisible, c'est-à-dire sont dotés d'une signification *. (POMIAN, Krzysztof. Collectionneurs, amateurs et curieux. Paris, Venise : $X V^{e}-X V I^{e}$ siècles. Paris : NRF Gallimard, 1987 , p. 42.)

[25]

DAVALLON, Jean.

«Gestes de mise en exposition ». In. DAVALLON, Jean (dir.). Claquemurer, pour ainsi dire, tout l'univers : la mise en exposition. Paris : Centre Georges Pompidou, 1986, p. 245.

[26]

SCHIELE,

op. cit., p. 239.

[27]

HAINARD, Jacques.

"La revanche du conservateur *. In. DESVALLÉES, «Exhiber ou démontrer?... *, op. cit, vol. 2, p. 403.

[28]

MAIRESSE,

op. cit, p. 144-145.

[29]

RIVIĖRE,

op. cit, p. 171.

[30]

MONTPETIT, Raymond. "Musées et muséologie : un champ de recherche dynamique en émergence *. In. LEMIEUX, Denise (dir.). Traité de la culture. Québec : Les Éditions de l'IORC / Les Presses de l'Université Laval, 2002, p. 82-84. 
[31]

BAUDRILLARD, Jean. Le système des objets. Paris : Gallimard, 1968, p. 111.

[32]

GASKELL, Ivan.

"Writing (and) Art History : Against Writing". Art Bulletin, vol. LXXVIII, $n^{\circ} 3$, septembre

1996, p. 403-406; et GASKELL, Ivan. «Magnanimity and Paranoia in the Big Bad Art World ". In. HAXTHAUSEN, Charles W. (dir.). The Two Art Histories : The Museum and the University. Williamstown (Mass.) : Sterling and Francis

Clark Art Institute, 2002,

p. 14-24.

[33]

SANDER, op. cit., p. 349.

\section{L'objet en fonction d'une discipline : l'histoire de l'art}

Il demeure encore quelque chose d'irréductible entre l'œuvre, le musée d'art et la discipline qui se préoccupe d'arts visuels. En effet, au-delà du prétexte de l'aura, de la quête d'authenticité, du trésor, ou du simple fétichisme (tellement réducteur), l'objet d'art constitue le fondement et le propos d'une discipline comme l'histoire de l'art, basée sur le matériel, le toucher, le saisissable du regard. Conservée, ou même disparue, l'œuvre demeure un noyau central vers lequel l'interprétation de l'historien de l'art est dirigée, peu importe les différentes possibilités épistémologiques (histoire, iconographie, sémiologie, sociologie, philosophie, anthropologie, etc.) dont il dispose. Ainsi, lorsque l'œuvre d'art est conservée dans l'institution muséale, elle ne constitue jamais un document, un témoin, un artefact ou une vague illustration aux yeux de l'historien de l'art. Elle constitue la pierre angulaire de la discipline même. De fait, l'approche holistique du musée favorisée par la muséologie, où l'objet est observé comme une entité globale, demeure une incongruité en regard d'une discipline comme l'histoire de l'art.

Dans cette perspective, la collection muséale ne représente pas seulement un "échelonnement quantitatif " ${ }^{\text {(3) }}$, pour reprendre l'expression de Jean Baudrillard, ou un vaste reposoir de moyens, mais autant d'occasions d'actualiser les connaissances, de redécouvrir l'art au-delà des caprices et des normes de l'histoire.

\section{Le musée, simple prestataire de services ?}

La recherche disciplinaire peut-elle être tout simplement remise entre les mains d'experts invités? Est-ce nécessaire pour l'institution d'employer, de façon permanente, des spécialistes tels que les conservateurs? Le musée pourrait-il constituer un simple lieu de référence, donnant accès aux œuvres selon les demandes de la communauté scientifique?

La délégation de la recherche muséale aux universités, par exemple, ne tient pas compte de la différence, encore bien actuelle, dans la pratique de l'histoire de l'art entre conservateurs et universitaires. Ivan Gaskell traite justement de cette problématique dans des textes publiés en 1996 et en 2002, où il met en évidence ces deux tendances : une première d'ordre livresque, liée à la théorie, qui se pratique à l'université; et une deuxième, plus pragmatique, liée à l'objet, aux collections, et qui se pratique au musée ${ }^{(22)}$. Ainsi, comme le note Sander, «le plus souvent, dans un musée, la recherche se préoccupe moins de théorisation ou de critique méthodologique que de questions en rapport direct avec les collections, ou du moins de questions concrètes s'appuyant sur l'étude comparative des objets $"{ }^{[33}$. 
Autrement dit, l'objet matériel constitué le centre d'attention du conservateur. Cette histoire de l'art est caractérisée par le développement de connaissances visuelles à la suite d'une proximité matérielle, constamment répétée, avec les objets d'art. Avec les années, le conservateur développe un "œil », une mémoire visuelle, $\mathrm{il}$ apprend à reconnaître les maniérismes des peintres, à effectuer des recoupements formels, à regrouper des œuvres sous le nom d'un même artiste et à procéder, de la sorte, aux attributions. Les attributions qui, rappelons-le, peuvent rapidement transformer la compréhension de l'œuvre d'un artiste et impliquer une révision complète de l'interprétation. De même, elles permettent de redécouvrir des artistes dont le travail était demeuré, jusque-là, dans le purgatoire des « inconnus». Pensons, notamment, au peintre Louis-Chrétien de Heer (1760-avant 1808), actif à Montréal et à Québec, dont la redécouverte de l'œuvre a été amorcée par Mario Béland, conservateur au Musée national des beaux-arts du Québec ${ }^{[34]}$. Le connaisseur se forme donc en présence des œuvres, dont le contact direct lui permet de se sensibiliser au caractère du peintre, à sa touche, aux détails répétés et qui lui sont propres.

En comparaison, à l'université, l'objet est davantage matière à interprétation et à théorisation. L'écriture précède bien souvent l'inspection matérielle de l'œuvre. Ces pratiques distinctes, et interdépendantes, ont leur importance spécifique puisqu'elles permettent, autant l'une que l'autre, le développement des savoirs dans la discipline. Mais leurs différences donnent à penser que le conservateur développe, par sa pratique et avec les années, des expertises différentes, essentielles tant à la recherche appliquée que disciplinaire.

\section{La légitimité de la parole : une lutte du conservateur pour son monopole?}

Dans un contexte de postmodernité, il semble que le musée comme le conservateur n'aient plus comme auparavant « la légitimité de proposer pour tous, un discours normatif global ${ }^{\text {[351. }}$. Toutefois, cela doit-il empêcher le conservateur de vouloir faire avancer l'état des connaissances? Il est illusoire de croire que le conservateur, par ses recherches multiples, parvienne à recréer l'histoire d'un objet d'art. Il base toujours ses recherches sur les documents qu'il connaît, qu'il a trouvés, puis qu'il interprète. Le conservateur, comme les autres acteurs du savoir (professeurs, universitaires ou, par exemple, commissaires professionnels), n'a d'autre choix que de se " compromettre" à la lumière de ses connaissances et de ses découvertes. C'est ainsi que le patrimoine artistique est actualisé et enrichi.

\section{[34]}

BÉLAND, Mario.

"Le capitaine François

Boucher *. Cap-aux-Diamants, no 70, été 2002, p. 59.

[35]

MONTPETIT,

"Musées et universités...", op. cit, p. 47. 
Comme ce fut le cas pour Le Grand Héritage (10 septembre 1984 au 13 janvier 1985) ou La peinture au Québec, 1820-1850. Nouveaux regards, nouvelles perspectives (16 octobre 1991 au 5 janvier 1992), deux expositions tenues au Musée du Québec (devenu le Musée national des beaux-arts du Québec en 2002) et dont les catalogues sont signés autant par des conservateurs que par des experts externes à un enjeu social », op. cit, p. 21.
Puisque l'histoire est relative, subordonnée à l'état de nos connaissances, pouvons-nous vraiment parler d'une lutte pour le monopole de la parole légitime? Pourtant, les connaissances sur l'objet, présentées sous la forme écrite dans l'exposition - comme les panneaux, les demi-panneaux, les chronologies, les cartels allongés et les cartels -, n'enlèvent rien, par exemple, à la vision personnelle que développe le visiteur au cours de la visite d'exposition. Capable d'imagination, d'émotions, d'opinions et de compréhension, il parvient, devant une œuvre qui l'intéresse, à adopter un comportement d'interaction. En fait, l'écrit dans une exposition présente nécessairement un point de vue (comment faire autrement ?), mais n'empêche pas le visiteur d'en adopter un autre, plus personnel. Le conservateur parvient, à tout le moins, à fournir les éléments historiques qui ont le mérite de répondre à certaines questions des visiteurs (quand cela a-t-il été fait, par qui, dans quel contexte, etc.). Il faut ainsi effectuer une claire distinction entre les connaissances relatives à l'œuvre et l'appropriation qu'en fait le public.

Par ailleurs, le conservateur correspond bien peu à la figure isolée qui "propose pour tous", étant en constante interrelation avec les autres professionnels du milieu (restaurateurs, professeurs, autres conservateurs, etc.). De fait, un musée comme le Musée national des beaux-arts du Québec fait appel à des comités d'experts externes pour évaluer l'intérêt des œuvres recommandées à l'acquisition par les conservateurs. De même, les découvertes des conservateurs, comme l'attribution des œuvres, nécessitent un consensus des spécialistes. Enfin, en ce qui concerne l'histoire de l'art au Québec, on remarque une grande collaboration et une mise en commun des connaissances entre l'expert invité et le conservateur en place ${ }^{[36]}$. Comme le remarque Laurier Lacroix, ces collaborations offrent l'avantage de ne pas « doubler les actions, et permettent de rationaliser les efforts " ${ }^{\left[{ }^{[37}\right.}$. En plus des dossiers conservés par le musée et des publications, ces collaborations permettent au savoir, acquis par le conservateur, de demeurer dans l'institution. 


\section{Le rôle de la recherche fondamentale au musée}

Les musées possèdent des collections qui recèlent encore une infinité de secrets. Considérer que la recherche sur les collections n'est utile que pour préparer des expositions, c'est ignorer les nombreuses et importantes contributions de la recherche muséale ${ }^{[38]}$.

La recherche au musée conduit à l'avancement de la discipline. Elle permet le développement de connaissances qui ont une incidence sur notre compréhension globale de l'histoire de l'art. Cependant, la recherche n'a pas pour unique objet l'érudition du conservateur, ou l'accumulation d'information qui n'intéresse que les experts. Elle permet au musée de parachever sa mission. En 1987, Jean Trudel affirmait que la recherche ne constitue pas une fonction isolée des autres fonctions muséales :

La recherche pourrait être considérée comme un investissement à long terme. C'est de la recherche que découlent les acquisitions judicieuses pour les collections et les musées, c'est de la recherche que découlent les projets d'expositions les plus intéressants, c'est de la recherche que découle la pertinence de l'information transmise au visiteur, c'est de la qualité de la recherche que découle la crédibilité d'un musée dans tous ses secteurs d'activité ${ }^{\text {(39). }}$.

$\underline{L}$ recherche disciplinaire permet ainsi au musée de soutenir adéquatement ses entreprises relatives tant à la médiation qu'au développement des collections.

En 1999, à la suite de la mise en ligne du Réseau canadien d'information sur le patrimoine (RCIP), Laurier Lacroix remarquait qu' " on a eu tendance à négliger les recherches sur les objets sélectionnés [...] les musées accumulent sans avoir toujours identifié le potentiel de ces objets. [...] Les dossiers sont souvent très maigres, et c'est un acte de foi qui est consenti envers l'objet lors de son acquisition ${ }^{[40]}$. " Pourtant, l'acquisition d'une œuvre implique l'utilisation de fonds importants : il faut la placer dans un espace à température contrôlée, veiller à sa sécurité et, si cela est nécessaire, la faire restaurer. Ces seules considérations d'ordre financier devraient nous rappeler la nécessité des recherches spécialisées afin de déterminer si l'œuvre est réellement nécessaire à l'institution.

Demandons-nous, en fait, quel intérêt aurait un important collectionneur à donner des œuvres à un musée afin qu'elles y soient oubliées? Non seulement le prestige de l'institution, mais également la renommée de son personnel spécialisé, encouragent les donations, lesquelles sont, d'ailleurs, le principal moyen d'acquisition de nombreuses institutions. La figure du conservateur, telle qu'esquissée par Luc Benoist en 1960, trouve toute sa pertinence :

\section{[38]}

MCGILLIVRAY, Bruce W. «La recherche muséale : évidence ou contradiction dans les termes?». Muse, vol. IX, $n^{\circ} 2$, été / automne 1991, p. 70.[39] TRUDEL, op. cit, p. 12. [40] LACROIX, Laurier. "Collectionner, un enjeu pour le XXle siècle *. Muse. vol. XVII, $n^{\circ} 2,1999$, p. 8. 
[41]

Cité dans RASSE, op. cit, p.14

[42]

Créé par le gouvernement du Québec, le musée ouvre ses portes en 1933, à Québec. II abrite alors les collections d'arts, de sciences naturelles (départies en 1962), ainsi que les Archives de la province (déménagées en 1979). La vocation de musée d'art est ensuite confirmée en 1983, lorsque le musée devient une société d'État. II s'agit alors de la seule institution muséale nationale ayant pour mandat de couvrir toute la production artistique québécoise, du XVIle siècle à nos jours. Après le nom de Musée de la

Province (1933) et de celui de Musée du Québec (1961), l'institution a changé de dénomination en 2002 pour celle de Musée national des beaux-arts du Québec.

[43]

Exposition Rodin à Québec, présentée au musée du 4 juin au 13 septembre 1998.

À ce sujet, on dit lire : PORTER, John R. «Rodin, Laliberté et les autres : réalités et perception d'un musée d'art". Annales d'histoire de l'art canadien, vol. XX, nos 1-2, 1999, p. 204-221.

[44]

Québec, Musée national des beaux-arts du Québec.

Plan stratégique 2005-2008.

Québec : Musée national des beaux-arts du Québec, 2005, p. 20.

[45]

Comme l'exposition Antoine Plamondon (1804-1895). Jalons d'un parcours artistique, organisée par John R. Porter (directeur général) et Mario Béland (conservateur de l'art ancien, 1850-1900), présentée au Musée national des beaux-arts du Québec du 24 novembre 2005 au 9 avril 2006. L'exposition est également itinérante et sera accueillie, jusqu'en 2008, par différents musées canadiens.
Un érudit imbattable sur les noms et les dates, un spécialiste del'histoire de l'art et des civilisations, un expert qui sache évaluer l'authenticité des objets qui lui sont proposés à l'examen ou à l'achat, un financier au courant de la cote de chaque artiste, [...] un diplomate qui cultive de belles relations pour obtenir des amateurs les dons ou legs sans lesquels son musée ne saurait s'enrichir ${ }^{[41]}$.

Le conservateur, spécialiste disciplinaire, permet ainsi le rayonnement et le développement des collections du musée, lesquelles nécessitent qu'on les éclaire et qu'on les fasse connaître.

\section{En guise de conclusion : public et recherche irréconciliables?}

L'examen d'un cas particulier, celui du Musée national des beaux-arts du Québec ${ }^{[42]}$, rend compte du fait que la recherche ne constitue pas un obstacle au travail de médiation du musée et que, réciproquement, la médiation n'implique pas la dissolution de la recherche disciplinaire au musée. De fait, l'institution a réalisé des expositions prodigieuses ces dernières années afin d'attirer un large public. On pense par exemple à l'exposition Rodin à Québec ${ }^{[43]}$, qui a eu un effet très remarqué par ses retombées économiques sur la ville de Québec en 1998, ou aux Grands Prix du tourisme québécois récoltés par l'institution en 2005 et 2006. Cet intérêt pour le public n'a pourtant pas signifié un changement complet du musée. Comme l'indique le Plan stratégique 2005-2008, «inscrire le MNBAQ[Musée national des beaux-arts du Québec] comme un lieu de recherche de pointe en art québécois " " ${ }^{[4]}$ constitue toujours l'un des objectifs poursuivis par les dirigeants, tant par la diffusion des recherches et la multiplication des partenariats que par le soutien accordé à la recherche effectuée à l'intérieur de l'enceinte muséale.

De fait, les conservateurs réalisent des expositions, tant permanentes que temporaires ${ }^{[45]}$, où sont mises en évidence les œuvres de la collection. Ils ajoutent même des volets québécois à des expositions à grand déploiement, comme ce fut le cas dans Copyright Rubens ${ }^{[46]}$, ou organisent des expositions satellites, comme Laliberté et Rodin présentée en même temps que l'exposition Rodin à Québec. Plus encore, les dirigeants ont opté pour un équilibre dans le travail des conservateurs, le temps étant réparti également entre les expositions et le développement des collections. Ainsi, les conservateurs de ce musée, qui sont tous des spécialistes en histoire de l'art québécois, doivent 
procéder à la documentation et au développement de la collection dont ils ont la charge. La recherche sur les objets, qui ne constitue pas un strict travail de catalogage, permet l'actualisation des dossiers d'œuvres, la préparation d'expositions et l'élaboration d'études en vue de nouvelles acquisitions.

Enfin, notons le travail de diffusion du musée, tant par la création et l'organisation d'expositions ${ }^{[47]}$ que par ses nombreuses publications ${ }^{[48]}$ portant sur l'art au Québec historique. En soutenant les projets des conservateurs, de même que ceux des commissaires invités et des divers partenaires de recherche, le musée est devenu un catalyseur essentiel pour la reconnaissance publique de la richesse du patrimoine québécois ainsi que pour l'avancement des connaissances disciplinaires.

Par ces initiatives multiples, le Musée national des beaux-arts du Québec peut sembler porter des actes antinomiques à notre réalité contemporaine. Pourtant, c'est ce qui lui permet de se démarquer dans le champ muséal. Finalement, le Musée national des beaux-arts du Québec prouve, par ses actions et ses prises de position, qu'un musée peut encore être un lieu de savoir, d'expertise et de recherche, tout en s'adaptant aux exigences des publics. Rubens.

\section{[47]}

Pensons, par exemple, en 2002-2003, à l'exposition Suzor-Côté, lumière et matière, dont le commissaire était Laurier Lacroix (professeur à l'Université du Québec à Montréal) et la coordonnatrice, Michèle Grandbois (conservatrice de l'art moderne, 1900-1950).

\section{[48]}

À titre d'exemples : BÉLAND, Mario (dir.). La peinture au Québec : 1820-1850. Nouveaux regards, nouvelles perspectives (cat). Québec : Musée du Québec / Les Publications du Québec, 1991, 605 p.; KAREL, David. Dictionnaire des artistes de langue française en Amérique du Nord. Québec : Musée du Québec / Les Presses de l'Université Laval, 1992, 962 p.; DROUIN, Daniel (dir.). Louis-Philippe Hébert (cat). Québec / Montréal : Musée du Québec / Musée des beauxarts de Montréal, 2001, 413 p.; BÉLAND, Mario (dir.). Questions de sculpture ancienne : hommage à Gérard Lavallée. Québec : Musée du Québec, 2003, 53 p.; PORTER, John R. et Mario BÉLAND. Antoine Plamondon (1804-1895). Jalons d'un parcours artistique (cat.). Québec : Musée national des beaux-arts du Québec, $2005,111 \mathrm{p}$. 


\section{Summary}

\section{The Role of Disciplinary Knowledge in Museum Research}

[translated by Victoria Surtees]

From displaying objects to displaying social activity, museums in the twentieth century have witnessed a reappraisal of their distinct activities. Marketing, technological development as well as competitive positioning among the cultural industry transformed the museum space into a more user friendly and event oriented field.

Closely linked to a sharp public awareness and to culture democratization, museum modernization has had many obvious beneficial effects. It is however important to point out that this conversion created a new conundrum. With human and economic resources and strategy development mainly invested in attracting more visitors, the disciplinary knowledge in museum research seems less of a priority.

Taking into account its historical transformation, the museum as a place of learning has probably given way to the museum as a place of mediation. In fact, the research previously carried out within their walls is largely being replaced by out-sourcing and the purchase of blockbuster exhibits. In this context, how can a museum's research still prove essential, even necessary to the institution? What are its defining features and who is involved?

Firstly, we will describe the historical foundations of the museum, and how its origins in the age of Enlightenment explain its role as a place of disciplinary knowledge, conserving both the physical and intellectual parts of its collection. Secondly, we will describe the museum's postmodern transformation and how the museum, in fact, occupies an increasingly recognized place in the cultural industry, presenting itself as a cultural past time, catering to the public, and aiming at profitability. We will be able to demonstrate how out-sourcing can address some immediate needs, when the curator is tied up managing exhibits, and be advantageous to the museum. However, we will also see that when used on a regular basis, it can lead to the loss of internal knowledge within the institution, reducing to a simple place of reference. Similarly, for an art museum, there is a crucial difference between object research, undertaken in a museum, and theoretical research, undertaken in a university. In general, ascription especially implies the development of specific knowledge gleaned from direct observation, de visu, and from the comparative study of objects. These skills, which the conservationist develops through knowledge of 
his collection, enrich both the discipline as well as the museum. In fact, rather than claiming that its research is the sole legitimate source of new knowledge, or gathering facts that appeal to experts alone, a museum performs research in order to achieve its mission, as much through its exhibits and educational programs as through its collections. A museum's disciplinary research allows the reanimation of objects' memories and the communication of pertinent information to visitors; it means that documented objects will be exhibited and that the acquisition of new objects will not be undertaken blindly. We believe that a balance between the democratization of the arts, mediation, and research is possible in Quebec, as was demonstrated by our personal experiences at the Musée national des beaux-arts du Québec. 